\title{
THE LACZKOVICH-KOMJÁTH PROPERTY FOR COANALYTIC EQUIVALENCE RELATIONS
}

\author{
SU GAO, STEVE JACKSON, AND VINCENT KIEFTENBELD
}

\begin{abstract}
Let $E$ be a coanalytic equivalence relation on a Polish space $X$ and $\left(A_{n}\right)_{n \in \omega}$ a sequence of analytic subsets of $X$. We prove that if $\lim _{\sup _{n \in K}} A_{n}$ meets uncountably many $E$-equivalence classes for every $K \in[\omega]^{\omega}$, then there exists a $K \in[\omega]^{\omega}$ such that $\bigcap_{n \in K} A_{n}$ contains a perfect set of pairwise $E$-inequivalent elements.
\end{abstract}

§1. Introduction. Let $\left(A_{n}\right)_{n \in \omega}$ be a sequence of sets and $K \in[\omega]^{\omega}$ an infinite subset of $\omega$. The limit superior $\lim _{\sup _{n \in K}} A_{n}$ is the set of all elements which belong to $A_{n}$ for infinitely many $n \in K$. Laczkovich [6] showed that for every sequence $\left(A_{n}\right)_{n \in \omega}$ of Borel sets in a Polish space, if $\lim _{\sup _{n \in K}} A_{n}$ is uncountable for every $K \in[\omega]^{\omega}$, then there exists a $K \in[\omega]^{\omega}$ such that $\bigcap_{n \in K} A_{n}$ is uncountable. Komjáth [5] generalized this result to the case where the sets $\left(A_{n}\right)_{n \in \omega}$ are analytic. Note that by the perfect set property of analytic sets, if $\bigcap_{n \in K} A_{n}$ is uncountable, then it contains a perfect set.

Balcerzak and Głąb [1] extended these results to $F_{\sigma}$ equivalence relations in the following way.

Definition. An equivalence relation $E$ on a Polish space $X$ has the LaczkovichKomjáth property if for every sequence $\left(A_{n}\right)_{n \in \omega}$ of analytic subsets of $X$ such that $\lim \sup _{n \in K} A_{n}$ meets uncountably many $E$-equivalence classes for every $K \in[\omega]^{\omega}$, there exists a $K \in[\omega]^{\omega}$ such that $\bigcap_{n \in K} A_{n}$ contains a perfect set of pairwise $E$ inequivalent elements.

In this terminology, Komjath has shown that the identity relation $=$ has the Laczkovich-Komjáth property. Balcerzak and Głąb [1] proved that every $F_{\sigma}$ equivalence relation has the Laczkovich-Komjáth property. In this paper, we generalize this to coanalytic equivalence relations.

THEOREM 1. Every coanalytic equivalence relation on a Polish space has the Laczkovich-Komjáth property.

Received August 14, 2009.

2000 Mathematics Subject Classification. Primary 03E15, 54H05; Secondary 28A05.

Key words and phrases. Limit superior of a sequence of sets, coanalytic equivalence relations, Laczkovich-Komjáth property.

The authors acknowledge the support of their research by US NSF grant DMS-0901853. The first author also acknowledges support by US NSF grant DMS-0501039. 
A fundamental result on coanalytic equivalence relations is Silver's theorem: a coanalytic equivalence relation either has only countably many equivalence classes, or else there exists a perfect set of pairwise inequivalent elements. Silver's original proof [9] used forcing. Harrington (unpublished) later gave a simpler (forcing) proof using effective descriptive set theory, which nowadays is usually cast in terms of the Gandy-Harrington topology. We will use similar methods and assume familiarity with effective descriptive set theory throughout the paper.

An introduction to effective descriptive set theory is given in [7], where the reader can also find the topological version of Harrington's proof. The review in [4] provides details on the Gandy-Harrington topology and strong Choquet games. Instead of strong Choquet games, we will make use of the set of low elements, which is a Polish space in the Gandy-Harrington topology. We will summarize the technical facts we use later on. Further details can be found in [2], which also provides another source on effective descriptive set theory.

This paper is organized as follows. In Section 2 we review a well-known coding mechanism for $\Pi_{1}^{1}$ and $\Delta_{1}^{1}$ sets, mainly to fix notation and establish the uniformity of a diagonal intersection operator. In Section 3, we provide details on canonical cofinal sequences as developed in [3]. We use these sequences in Section 4 to prove our main technical result. Finally, we derive our main theorem in Section 5, where we also derive a parametric version of the theorem, as was done by Balcerzak and Głąb [1].

§2. Coding $\Pi_{1}^{1}$ and $\Delta_{1}^{1}$ sets. In this section we review a well-known coding mechanism for $\Pi_{1}^{1}$ and $\Delta_{1}^{1}$ sets, mainly to fix notation. A good introduction can be found in [4, Section 3.2], where the notion of uniformity is also discussed. We will need the uniformity of a diagonal intersection operation. Since this operation is not canonical, we provide a little more of the details.

A product space is any $X=X_{0} \times \cdots \times X_{n}$ (with the product topology), where each factor is either $\omega$ or $\omega^{\omega}$. For every product space $X$ there is a $U^{X} \subseteq \omega \times X$ such that $U^{X} \in \Pi_{1}^{1}$ and for any $A \subseteq X, A \in \Pi_{1}^{1}$ iff $\exists n\left(A=U_{n}^{X}\right)$. Such a set $U^{X}$ is called a universal $\Pi_{1}^{1}$ set. A $\Pi_{1}^{1}$ code for $A \subseteq X$ is any $n \in \omega$ such that $A=U_{n}^{X}$.

There exists a collection $\left\{U^{X}\right\}$ of universal $\Pi_{1}^{1}$ sets with the following additional property: for any $m \in \omega$ and any product space $X$ there is a recursive function $S^{m, X}: \omega^{m+1} \rightarrow \omega$ such that

$$
\left(e, k_{1}, \ldots, k_{m}, x\right) \in U^{\omega^{m} \times X} \Leftrightarrow\left(S^{m, X}\left(e, k_{1}, \ldots, k_{m}\right), x\right) \in U^{X} .
$$

Such a collection is called a good universal system. For the rest of this paper, fix a good universal system $\left\{U^{X}\right\}$ for $\Pi_{1}^{1}$. This good universal system can be used to code $\Delta_{1}^{1}$ subsets, which we now describe. This coding is always relative to a particular product space $X$. When there is no danger of confusion, we will drop the superscript in $U^{X}$. For every $k \in \omega$, fix a recursive bijection $\left(n_{0}, \ldots, n_{k-1}\right) \mapsto\left\langle n_{0}, \ldots, n_{k-1}\right\rangle$ between $\omega^{k}$ and $\omega$, and denote the recursive inverse map by $n \mapsto\left((n)_{0}, \ldots,(n)_{k-1}\right)$. Define

$$
\begin{aligned}
& (\langle m, n\rangle, x) \in U_{0} \Leftrightarrow(m, x) \in U, \\
& (\langle m, n\rangle, x) \in U_{1} \Leftrightarrow(n, x) \in U .
\end{aligned}
$$


Then $U_{0}, U_{1} \in \Pi_{1}^{1}$. By the reduction property for $\Pi_{1}^{1}$ sets, there are $\Pi_{1}^{1}$ sets $U_{0}^{*}, U_{1}^{*} \subseteq \omega \times X$ such that $U_{0}^{*} \cup U_{1}^{*}=U_{0} \cup U_{1}$ and $U_{0}^{*} \cap U_{1}^{*}=\emptyset$. Let $P=U_{0}^{*}$ and $S=(\omega \times X) \backslash U_{1}^{*}$. Define

$$
\langle m, n\rangle \in C \Leftrightarrow \forall x \in X\left((\langle m, n\rangle, x) \in U_{0}^{*} \vee(\langle m, n\rangle, x) \in U_{1}^{*}\right) .
$$

Then $C \in \Pi_{1}^{1}$. For all $n \in C$,

$$
P_{n}=S_{n}:=D_{n}
$$

A $\Delta_{1}^{1}$ code for $A \subseteq X$ is any $n \in C$ such that $A=D_{n}$. In that case, $(n)_{0}$ is a $\Pi_{1}^{1}$ code for $A$ and $(n)_{1}$ is a $\Pi_{1}^{1}$ code for $X \backslash A$. Conversely, if $m, n \in \omega$ are $\Pi_{1}^{1}$ codes for $A$ and $X \backslash A$, respectively, then $\langle m, n\rangle$ is a $\Delta_{1}^{1}$ code for $A$. It is important that the set $C$ of $\Delta_{1}^{1}$ codes is $\Pi_{1}^{1}$ and that operations hold effectively in the codes, in the following way.

Example. Given $\Delta_{1}^{1}$ codes $m, n \in C$ for $A, B \subseteq X$, we can effectively compute a $\Delta_{1}^{1}$ code for $A \backslash B$. To see this, define

$$
\begin{aligned}
& (m, n, x) \in Z_{0} \Leftrightarrow x \in D_{m} \wedge x \notin D_{n}, \\
& (m, n, x) \in Z_{1} \Leftrightarrow x \notin D_{m} \vee x \in D_{n} .
\end{aligned}
$$

Clearly, $Z_{0}, Z_{1} \in \Pi_{1}^{1}$. Let $e_{0}, e_{1}$ be their respective $\Pi_{1}^{1}$ codes. Then for $i=0,1$,

$$
(m, n, x) \in Z_{i} \Leftrightarrow\left(e_{i}, m, n, x\right) \in U^{\omega^{2} \times X} \Leftrightarrow\left(S^{2, X}\left(e_{i}, m, n\right), x\right) \in U^{X} .
$$

Also, $Z_{0}=\left(\omega^{2} \times X\right) \backslash Z_{1}$. Thus,

$$
\left\langle S^{2, X}\left(e_{0}, m, n\right), S^{2, X}\left(e_{1}, m, n\right)\right\rangle
$$

is a $\Delta_{1}^{1}$ code for $A \backslash B$.

Similar uniformities hold for all basic set-theoretic operations. We will need the uniformity of a diagonal intersection operator, which we define next. Recall that when $H, K \in[\omega]^{\omega}, H \subseteq^{*} K$ denotes that $H$ is almost contained in $K$, i.e., $K \backslash H$ is finite.

Definition. For a (finite or infinite) sequence $\left(K_{n}\right)$ of infinite subsets of $\omega$ with $K_{n} \subseteq^{*} K_{m}$ for $n>m$, define $\triangle K_{n}$ by $m \in \triangle K_{n}$ iff there exists $m_{0}<m_{1}<\cdots<$ $m_{k}=m$ such that $m_{0}$ is the least element of $K_{0}, m_{1}$ is the least element of $K_{0} \cap K_{1}$ such that $m_{1}>m_{0}, \ldots, m_{k}$ is the least element of $K_{0} \cap \cdots \cap K_{k}$ such that $m_{k}>m_{k-1}$.

Note that $\triangle K_{n} \subseteq^{*} K_{m}$ for all $m$. To obtain the desired uniformity for this diagonal intersection operation, we need to assume that the sequence of $\Delta_{1}^{1}$ codes for $\left(K_{n}\right)$ is effective. One way to formalize this is to let $n \in C^{*}$ iff

1. $n \in C^{\omega}$,

2. $D_{n}^{\omega}$ is infinite,

3. $\forall m\left(m \in D_{n}^{\omega} \Rightarrow(m)_{1} \in C^{\omega}\right)$, and

4. $\forall i \exists ! m\left(m \in D_{n}^{\omega} \wedge(m)_{0}=i\right)$.

Informally, $n \in C^{*}$ iff $n$ is a $\Delta_{1}^{1}$ code for an infinite subset of $\omega$ of the form $\left\{\left\langle i, n_{i}\right\rangle: i \in \omega, n_{i} \in C\right\}$. Clearly, $C^{*} \in \Pi_{1}^{1}$.

Lemma 2. There is a function Diag: $\omega \rightarrow \omega$ which is $\Delta_{1}^{1}$ on $C^{*}$ such that whenever $n \in C^{*}$ is a code for an infinite $\Delta_{1}^{1}$ subset $\left\{\left\langle i, n_{i}\right\rangle: i \in \omega, n_{i} \in C\right\}$ of $\omega, \operatorname{Diag}(n)$ is a $\Delta_{1}^{1}$ code for $\triangle D_{n_{i}}^{\omega}$. 
Proof. It suffices to find $\Pi_{1}^{1}$ codes $e_{0}$ and $e_{1}$ for $\triangle D_{n_{i}}^{\omega}$ and $\omega \backslash \triangle D_{n_{i}}^{\omega}$, respectively, because $\left\langle e_{0}, e_{1}\right\rangle$ will then be a $\Delta_{1}^{1}$ code for $\triangle D_{n_{i}}^{\omega}$. We need the following three facts:

1. There is a recursive function $u: \omega \rightarrow \omega$ such that whenever $n=\left\langle n_{0}, \ldots, n_{k}\right\rangle$ is a finite sequence of $\Delta_{1}^{1}$ codes, $u(n)$ is a $\Delta_{1}^{1}$ code for $D_{n_{0}}^{\omega} \cap \cdots \cap D_{n_{k}}^{\omega}$.

2. There is a $\Delta_{1}^{1}$ on the codes function $i: \omega \times \omega \rightarrow \omega$ such that whenever $n \in C^{*}$, $i(n, j)$ is the (unique) $m \in \omega$ such that $\langle j, m\rangle \in D_{n}^{\omega}$.

3. There is a $\Delta_{1}^{1}$ on the codes function $\mu: \omega \times \omega \rightarrow \omega$ such that whenever $n$ is a $\Delta_{1}^{1}$ code for an infinite subset of $\omega, \mu(n, j)$ is the least element of $D_{n}^{\omega}$ greater than or equal to $j$.

Now define

$$
\begin{aligned}
&(n, m) \in Z_{0} \Leftrightarrow n \in C^{*} \wedge \exists\left\langle m_{0}, \ldots, m_{k}\right\rangle\left(m_{0}<\cdots<m_{k} \wedge m_{k}=m \wedge\right. \\
& m_{0}=\mu(u(\langle i(n, 0)\rangle), 0) \wedge m_{1}=\mu\left(u(\langle i(n, 0), i(n, 1)\rangle), m_{0}+1\right) \wedge \\
&\left.\cdots \wedge m_{k}=\mu\left(u(\langle i(n, 0), \ldots, i(n, k)\rangle), m_{k-1}+1\right)\right) .
\end{aligned}
$$

Then $Z_{0} \in \Pi_{1}^{1}$. Pick a $\Pi_{1}^{1}$ code $e_{0}$ for $Z_{0}$. Similarly, we can write down a $\Pi_{1}^{1}$ definition for $Z_{1}=C^{*} \backslash Z_{0}$ and pick a $\Pi_{1}^{1}$ code $e_{1}$. The rest of the argument is as in the example.

Now that we have established the uniformity of this diagonal intersection operator, we will use it implicitly. Finally, for codes $h, k \in C^{\omega}$, we write $h \subseteq^{*} k$ iff the set coded by $h$ is almost contained in the set coded by $k$. Writing out the definitions, we see that $h \subseteq^{*} k$ is $\Delta_{1}^{1}$ on the set $C^{\omega}$ of codes.

§3. Canonical cofinal sequences. For $w \in 2^{\omega}$, define a binary relation $<_{w}$ on a subset of $\omega$ by

$$
m<{ }_{w} n \Leftrightarrow w(\langle m, n\rangle)=1 .
$$

The domain of $<_{w}$ is the set

$$
\operatorname{dom}\left(<_{w}\right)=\left\{n \in \omega: \exists m \in \omega\left(m<_{w} n \text { or } n<_{w} m\right)\right\} .
$$

Let LO denote the set of all $w \in 2^{\omega}$ such that $<_{w}$ is a linear order, and let LO* denote the set of all $w \in \mathrm{LO}$ such that $<_{w}$ has a least element and every $n \in \operatorname{dom}\left(<_{w}\right)$ has an immediate successor $n_{<_{w}}^{+}$. For $w \in \mathrm{LO}$, let $\left|<_{w}\right|$ denote the order type of $<_{w}$. The next lemma shows that in a uniform way, we can effectively obtain a canonical cofinal sequence in $<_{w}$ given $w \in \mathrm{LO}^{*}$.

Lemma 3 (Gao-Jackson-Laczkovich-Mauldin [3]). There is a $\Delta_{1}^{1}$ function

$$
\text { Cof }:\left\{(w, n, j) \in \mathrm{LO}^{*} \times \omega^{2}: n \in \operatorname{dom}\left(<_{w}\right)\right\} \rightarrow \omega
$$

such that

1. if $w \in \mathrm{LO}^{*}, n \in \operatorname{dom}\left(<_{w}\right)$ and $j \in \omega$, then $\operatorname{Cof}(w, n, j) \in \operatorname{dom}\left(<_{w}\right)$ and $\operatorname{Cof}(w, n, j)<_{w} n$, unless $n$ is the $<_{w}$-least element;

2. if $w \in \mathrm{LO}^{*}$ and $n \in \operatorname{dom}\left(<_{w}\right)$ has an immediate predecessor in $<_{w}$, then $\operatorname{Cof}(w, n, j)_{w}^{+}=n$ for all $j \in \omega$;

3. if $w \in \mathrm{LO}^{*}, n \in \operatorname{dom}\left(<_{w}\right)$ is not $<_{w}$-least and $n$ does not have an immediate predecessor in $<_{w}$, then 
(a) if $j<j^{\prime}$, then $\operatorname{Cof}(w, n, j)<{ }_{w} \operatorname{Cof}\left(w, n, j^{\prime}\right)$, and

(b) for any $q \in \operatorname{dom}\left(<_{w}\right)$ with $q<_{w} n$ there is a $j \in \omega$ such that $q<_{w}$ $\operatorname{Cof}(w, n, j)$.

We also need a variation of this lemma for $\Pi_{1}^{1}$ norms, whose proof uses the same ideas. Recall that a $\Pi_{1}^{1}$-norm on a pointset $P \in \Pi_{1}^{1}$ is a function $\varphi$ from $P$ into the ordinals On such that there exist binary relations $<_{\varphi}^{*}$ and $\leq_{\varphi}^{*}$ in $\Pi_{1}^{1}$ with the following properties:

$$
\begin{aligned}
& x \leq_{\varphi}^{*} y \Leftrightarrow P(x) \wedge(\neg P(y) \vee \varphi(x) \leq \varphi(y)), \\
& x<_{\varphi}^{*} y \Leftrightarrow P(x) \wedge(\neg P(y) \vee \varphi(x)<\varphi(y)) .
\end{aligned}
$$

Recall that WO denotes the set of all $w \in \mathrm{LO}$ such that $<_{w}$ is a well-order. Every $\Pi_{1}^{1}$ set $P \subseteq \omega$ admits a $\Pi_{1}^{1}$-norm $\varphi: P \rightarrow \omega_{1}^{\mathrm{CK}}$, where

$$
\omega_{1}^{\mathrm{CK}}=\sup \left\{\left|<_{w}\right|: w \in \mathrm{WO} \text { is recursive }\right\},
$$

see for example [8, Section 4B].

Lemma 4. Let $\varphi$ be a $\Pi_{1}^{1}$-norm on a $\Pi_{1}^{1}$ set $P \subseteq \omega$. There is a $\Pi_{1}^{1}$ function Cof : $\omega \rightarrow \omega$ such that

1. for all $j \in \omega, \operatorname{Cof}(j) \in P$;

2. if $j<j^{\prime}$, then $\operatorname{Cof}(j)<_{\varphi}^{*} \operatorname{Cof}\left(j^{\prime}\right)$ unless $\operatorname{Cof}(j)$ is $<_{\varphi}$-maximal;

3. for any $q \in P$, there is a $j \in \omega$ such that $q<_{\varphi}^{*} \operatorname{Cof}(j)$ unless $q$ is $<_{\varphi}$-maximal.

Proof. We define the function Cof by induction on $j$. Let $p_{0}=\operatorname{Cof}(0)$ be the least integer in $P$. Assume we have defined $p_{j}=\operatorname{Cof}(j)$. If $p_{j}$ is $<_{\varphi}$-maximal, let $p_{j+1}=p_{j}$. Otherwise, let $p_{j+1}=\operatorname{Cof}(j+1)$ be the smallest integer in $P$ such that $p_{j}<p_{j+1}$ and $p_{j}<_{\varphi}^{*} p_{j+1}$. Since $n=p_{j+1}$ iff $n \in P$ and $p_{j}<n$ and $p_{j}<_{\varphi}^{*} n$ and $\forall m\left(p_{j}<m<n \Rightarrow m \leq_{\varphi}^{*} p_{j}\right)$, this defines a $\Pi_{1}^{1}$ function. To see that $(3)$ holds, let $q \in P$ be a nonmaximal element. Since the sequence $\left(p_{j}\right)_{j \in \omega}$ is strictly increasing in the natural order $<$ on $\omega$, there is a least integer $j$ such that $p_{j} \leq q<p_{j+1}$. Because $p_{j+1}$ is the least integer larger than $p_{j}$ such that $p_{j}<_{\varphi}^{*} p_{j+1}$, we cannot have $p_{j}<_{\varphi}^{*} q$. Hence, $q \leq_{\varphi}^{*} p_{j}<_{\varphi}^{*} p_{j+1}$.

$\S 4$. A completely good pair. Suppose $E$ is a $\Pi_{1}^{1}$ equivalence relation on $\omega^{\omega}$. A key idea in Harrington's proof of Silver's dichotomy is to consider the set

$$
W=\left\{x \in \omega^{\omega}: \text { there is no } \Delta_{1}^{1} \text { set } D \text { such that } x \in D \subseteq[x]_{E}\right\} .
$$

A computation shows that $W$ is $\Sigma_{1}^{1}$. Moreover, when $E$ has uncountably many equivalence classes, $W \neq \emptyset$ and every nonempty $\Sigma_{1}^{1}$ subset $X \subseteq W$ meets uncountably many $E$-equivalence classes. In fact, a nonempty $\Sigma_{1}^{1}$ subset $X \subseteq \omega^{\omega}$ meets uncountably many $E$-equivalence classes iff $X \cap W \neq \emptyset$.

We will establish the following corresponding result in our context.

Proposition 5. Let $E$ be a $\Pi_{1}^{1}$ equivalence relation on $\omega^{\omega}$ and $\left(A_{n}\right)_{n \in \omega}$ a sequence of uniformly $\Sigma_{1}^{1}$ subsets of $\omega^{\omega}$. If $\lim \sup _{n \in K} A_{n}$ meets uncountably many $E$ equivalence classes for every $K \in[\omega]^{\omega}$, then there exists a nonempty $\Sigma_{1}^{1}$ set $V \subseteq \omega^{\omega}$ and a $\Delta_{1}^{1}$ set $H \in[\omega]^{\omega}$ such that for every nonempty $\Sigma_{1}^{1}$ set $X \subseteq V$ and every $\Delta_{1}^{1}$ set $K \in[H]^{\omega}$ the set $X \cap \lim \sup _{n \in K} A_{n}$ meets uncountably many E-equivalence classes. 
We call such a pair $(V, H)$ completely good. The rest of this section is devoted to the proof of Proposition 5 and a further refinement. In contrast with Harrington's proof, we need a recursive construction of transfinite length, in which we remove all possible 'bad pairs' one by one.

Definition. We say that $n=\langle y, k\rangle \in \omega$ is a bad pair if the following properties hold:

1. $y \in C^{\omega^{\omega}}$ and $k \in C^{\omega}$,

2. $D_{k}^{\omega} \in[\omega]^{\omega}$, and

3. $D_{y}^{\omega^{\omega}} \cap \lim \sup _{n \in D_{k}^{\omega}} A_{n}$ meets only countably many $E$-equivalence classes, i.e., $D_{y}^{\omega^{\omega}} \cap W \cap \lim \sup _{n \in D_{k}^{\omega}} A_{n}=\emptyset$.

It is clear from this definition that the set $P \subseteq \omega$ of all bad pairs is $\Pi_{1}^{1}$. Let $\varphi: P \rightarrow \omega_{1}^{\mathrm{CK}}$ be a $\Pi_{1}^{1}$-norm on $P$. Define a well-order on $P$ by

$$
m<{ }_{\varphi} n \Leftrightarrow \varphi(m)<\varphi(n) \vee(\varphi(m)=\varphi(n) \wedge m<n)
$$

and let $\leq_{\varphi}^{*}$ be the $\Pi_{1}^{1}$ relation given by

$$
m \leq_{\varphi}^{*} n \Leftrightarrow P(m) \wedge(\neg P(n) \vee \varphi(m) \leq \varphi(n)) .
$$

For the rest of the paper, let Cof : $\omega \rightarrow \omega$ be the $\Pi_{1}^{1}$ function related to $\varphi$ and $P$ as given by Lemma 4.

Denote by $C_{\infty}^{\omega}$ the set of all $n \in C^{\omega}$ such that $D_{n}^{\omega} \in[\omega]^{\omega}$. Then $C_{\infty}^{\omega}$ is $\Pi_{1}^{1}$. Given an $h \in C_{\infty}^{\omega}$, we define the next bad pair relative to $h$ to be the $<_{\varphi}$-least $\langle y, k\rangle \in P$ such that $k \subseteq^{*} h$. Set $R(h,\langle y, k\rangle)$ iff $\langle y, k\rangle$ is the next bad pair relative to $h$.

Lemma 6. The relation $R \subseteq \omega \times \omega$ is $\Pi_{1}^{1}$. Moreover, $R$ is a $\Delta_{1}^{1}$ function on the set $B=\left\{h \in \omega: h \in C_{\infty}^{\omega} \wedge \exists n(R(h, n))\right\}$.

Proof. We have $R(h,\langle y, k\rangle)$ iff

$$
h \in C_{\infty}^{\omega} \wedge\langle y, k\rangle \in P \wedge k \subseteq^{*} h \wedge \forall y^{\prime}, k^{\prime} \in \omega\left(\langle y, k\rangle \not_{\varphi}^{*}\left\langle y^{\prime}, k^{\prime}\right\rangle \Rightarrow k^{\prime} \nsubseteq^{*} h\right) .
$$

This is a $\Pi_{1}^{1}$ definition. If $R(h, n)$ holds, then $n$ is the unique such integer. Thus, for $h \in B, \neg R(h, n) \Leftrightarrow \exists m(R(h, m) \wedge n \neq m)$, which is $\Pi_{1}^{1}$. Hence, $R$ is $\Delta_{1}^{1}$ on $B . \quad-$ Similarly, given a $\Pi_{1}^{1}$ set $A \subseteq P$ we define the next bad pair in $A$ relative to $h$ to be the $<_{\varphi}$-least $\langle y, k\rangle \in A$ such that $k \subseteq^{*} h$. The corresponding version of Lemma 6 still holds.

Initial segments of the recursive construction can be coded by reals, as follows. Recall that $\mathrm{WO}_{\alpha}=\left\{w \in \mathrm{WO}:\left|<_{w}\right|=\alpha\right\}$ and for $\alpha<\omega_{1}^{\mathrm{CK}}$, we have $\mathrm{WO}_{\alpha} \in \Delta_{1}^{1}$.

Definition. Let $\alpha<\omega_{1}^{\mathrm{CK}}$. A real $z \in \omega^{\omega}$ is $\alpha$-adequate if $z=\langle w, v, h\rangle$, where $w \in 2^{\omega}, v \in \omega^{\omega}$, and $h \in \omega^{\omega}$, and the following conditions are satisfied:

1. $w \in \mathrm{WO}_{\alpha}$,

2. if $n \notin \operatorname{dom}\left(<_{w}\right)$, then $v(n)=h(n)=0$,

3. the $<_{w}$-least element is the $<_{\varphi}$-least element,

4. if $n \in \operatorname{dom}\left(<_{w}\right)$ is a $<_{w}$-successor ( $\operatorname{say} n=m_{<_{w}}^{+}$), then the following holds:

(a) $n=\langle y, k\rangle$ is the next bad pair relative to $h(m)$ such that $\langle y, k\rangle \notin$ $\operatorname{dom}\left(<_{w}\right)\lceil n$

(b) $v(n)$ is a canonical code for $D_{v(m)}^{\omega^{\omega}} \backslash D_{y}^{\omega^{\omega}}$,

(c) $h(n)=k$. 
5. if $n \in \operatorname{dom}\left(<_{w}\right)$ is a $<_{w}$-limit, then with $v^{\prime}$ the canonical code for

$$
\bigcap_{j \in \omega} D_{v(\operatorname{Cof}(w, n, j))}^{\omega^{\omega}}
$$

and $h^{\prime}$ the canonical code for $\triangle_{j \in \omega} D_{h(\operatorname{Cof}(w, n, j))}^{\omega}$, the following holds:

(a) $n=\langle y, k\rangle$ is the next pair relative to $h^{\prime}$ such that $\langle y, k\rangle \notin \operatorname{dom}\left(<_{w}\right)\lceil n$,

(b) $v(n)$ is the canonical code for $D_{v^{\prime}}^{\omega^{\omega}} \backslash D_{y}^{\omega^{\omega}}$, and

(c) $h(n)=k$.

Some comments on these conditions: (1) says that $z$ represents the construction up to stage $\alpha,(2)$ is needed only to ensure that there can be at most one $\alpha$-adequate real for every $\alpha<\omega_{1}^{\mathrm{CK}},(3),(4 \mathrm{a})$, and (5a) state that $<_{w}$ represents the order in which the bad pairs are picked in our construction and that we pick a new bad pair at each stage, and conditions $(4 \mathrm{~b}, \mathrm{c})$ and $(5 \mathrm{~b}, \mathrm{c})$ require $v(n)$ and $h(n)$ to be codes for the correct sets whenever $n \in \operatorname{dom}\left(<_{w}\right)$.

We call a real adequate if it is $\alpha$-adequate for some $\alpha<\omega_{1}^{\mathrm{CK}}$.

Lemma 7. The set of all adequate reals is $\Pi_{1}^{1}$.

Proof. Replace condition (1) above with condition (1) $w \in \mathrm{WO}_{<\omega_{1}^{\mathrm{CK}}}$, which is $\Pi_{1}^{1}$. Conditions (2) and (3) are arithmetical. For (4), $n$ is a $<_{w}$-successor, $n=(m)_{<_{w}}^{+}$, and $(4 \mathrm{~b}, \mathrm{c})$ are arithmetical predicates, while $(4 \mathrm{a})$ is $\Pi_{1}^{1}$. Thus, (4) is $\Pi_{1}^{1}$. Similarly, (5) is $\Pi_{1}^{1}$.

It is immediate from the definition of $\alpha$-adequate that for each $\alpha<\omega_{1}^{\mathrm{CK}}$, if there is an $\alpha$-adequate real, then this real is unique; denote it by $z_{\alpha}$.

Lemma 8. Every adequate real is $\Delta_{1}^{1}$.

Proof. Let $z_{\alpha}$ be $\alpha$-adequate for some fixed $\alpha<\omega_{1}^{\mathrm{CK}}$. Then $z=\langle w, v, h\rangle$ equals $z_{\alpha}$ iff $z$ satisfies conditions (1) through (5). The first 3 conditions are $\Delta_{1}^{1}$. Conditions (4) and (5) are $\Pi_{1}^{1}$, because (4a) and (5a) contain a predicate $R(n, h)$, i.e., $n$ is the next bad pair relative to $h$ (where $h=h(m)$ in 4 a and $h=h^{\prime}$ in 5a). However, since $z$ is given, we know that this $h$ is an element of $B=\{h \in \omega: h \in$ $\left.C_{\infty}^{\omega} \wedge \exists n(R(h, n))\right\}$. By Lemma $6, R$ is $\Delta_{1}^{1}$ on $B$. Thus, conditions (4) and (5) are $\Delta_{1}^{1}$ in this case.

Finally, we define $V \subseteq \omega^{\omega}$ and $H \in[\omega]^{\omega}$ as follows. Let $x \in V$ iff

$$
\forall z \in \Delta_{1}^{1}\left(z=\langle w, v, h\rangle \text { adequate } \Rightarrow \forall n\left(n \in \operatorname{dom}\left(<_{w}\right) \Rightarrow x \in D_{v(n)}^{\omega^{\omega}}\right)\right)
$$

and $n \in H$ iff

$$
\begin{aligned}
\exists z \in \Delta_{1}^{1}(z=\langle w, v, h\rangle & \text { is adequate } \wedge \\
& \left.\forall j \leq n\left(\operatorname{Cof}(j) \in \operatorname{dom}\left(<_{w}\right) \Rightarrow n \in \triangle_{j \leq n} h(\operatorname{Cof}(j))\right)\right) .
\end{aligned}
$$

Equivalently by Lemma $8, n \in H$ iff

$$
\begin{aligned}
& \forall z \in \Delta_{1}^{1}(z=\langle w, v, h\rangle \text { is adequate } \wedge \\
& \left.\qquad \forall j \leq n\left(\operatorname{Cof}(j) \in \operatorname{dom}\left(<_{w}\right) \Rightarrow n \in \triangle_{j \leq n} h(\operatorname{Cof}(j))\right)\right) .
\end{aligned}
$$

Lemma 9. $V \in \Sigma_{1}^{1}$ and $H \in \Delta_{1}^{1}$. Moreover, $V \neq \emptyset$ and $H \in[\omega]^{\omega}$. 
Proof. By Kleene's restricted quantification theorem (see for example [8, Theorem 4D.3]), $V \in \Sigma_{1}^{1}$. (Note: if the construction stops below $\omega_{1}^{\mathrm{CK}}$, then $V$ is actually $\Delta_{1}^{1}$ but we will not need that fact.) Similarly, the first definition of $H$ is $\Pi_{1}^{1}$ and the second definition is $\Sigma_{1}^{1}$. Therefore, $H \in[\omega]^{\omega}$ is $\Delta_{1}^{1}$. We show that $V \neq \emptyset$.

Suppose towards a contradiction that $V=\emptyset$. Then for every $x \in \omega^{\omega}$ there is an $\alpha<\omega_{1}^{\mathrm{CK}}$ and a $k \in \omega$ such that for $z_{\alpha}=\left\langle w_{\alpha}, v_{\alpha}, h_{\alpha}\right\rangle$, we have $k \in \operatorname{dom}\left(<_{w_{\alpha}}\right)$ and $x \notin D_{v_{\alpha}(k)}^{\omega^{\omega}}$. For $k \in \operatorname{dom}\left(<_{w_{\alpha}}\right)$, denote by $y_{\alpha}(k)$ the code for the set removed at that stage. By assumption,

$$
\omega^{\omega}=\bigcup_{\alpha<\omega_{1}^{C K}} \bigcup_{k \in \operatorname{dom}\left(<w_{\alpha}\right)} D_{y_{\alpha}(k)}^{\omega^{\omega}} .
$$

Since $H \subseteq^{*} D_{h_{\alpha}(k)}^{\omega}$ for every $k \in \operatorname{dom}\left(<_{w_{\alpha}}\right)$,

$$
\limsup _{n \in H} A_{n} \subseteq \limsup _{n \in D_{h_{\alpha}(k)}^{\omega}} A_{n} .
$$

In particular for every $k \in \operatorname{dom}\left(<_{w_{\alpha}}\right)$,

$$
D_{y_{\alpha}(k)}^{\omega^{\omega}} \cap \limsup _{n \in H} A_{n} \subseteq D_{y_{\alpha}(k)}^{\omega^{\omega}} \cap \limsup _{n \in D_{h_{\alpha}(k)}^{\omega}} A_{n} .
$$

Hence,

$$
\begin{aligned}
\limsup _{n \in H} A_{n} & =\bigcup_{\alpha<\omega_{1}^{\mathrm{CK}}} \bigcup_{k \in \operatorname{dom}\left(<_{w_{\alpha}}\right)} D_{y_{\alpha}(k)}^{\omega^{\omega}} \cap \limsup _{n \in H} A_{n} \\
& \subseteq \bigcup_{\alpha<\omega_{1}^{\mathrm{CK}}} \bigcup_{k \in \operatorname{dom}\left(<_{w_{\alpha}}\right)} D_{y_{\alpha}(k)}^{\omega^{\omega}} \cap \limsup _{n \in D_{h_{\alpha}(k)}^{\omega}} A_{n}
\end{aligned}
$$

meets only countably many $E$-equivalence classes, a contradiction. Thus, $V \neq \emptyset . \quad \dashv$ We now verify that the pair $(V, H)$ is indeed completely good. In the proof of the next lemma we use the following observation. Let $z=\langle w, v, h\rangle$ be an adequate real. If $m<_{w} n$, then $m, n \in P$ and $\varphi(m)<\varphi(n)$. This is the case, because whenever $\langle y, k\rangle$ is a bad pair such that $k \subseteq^{*} h(n)$, also $k \subseteq^{*} h(m)$, since $h(n) \subseteq^{*} h(m)$.

Lemma 10. If $X \subseteq V$ is a nonempty $\Sigma_{1}^{1}$ set and $K \in[H]^{\omega}$ a $\Delta_{1}^{1}$ set, then $X \cap$ $\lim \sup _{n \in K} A_{n}$ meets uncountably many E-classes.

Proof. Suppose $X \cap \lim \sup _{n \in K} A_{n}$ meets only countably many $E$-equivalence classes, i.e., $X \cap \lim \sup _{n \in K} A_{n} \cap W=\emptyset$. By $\Sigma_{1}^{1}$-separation, there is a $\Delta_{1}^{1}$ set $Y \subseteq \omega^{\omega}$ such that $X \subseteq Y$ and $Y \cap \lim \sup _{n \in K} A_{n} \cap W=\emptyset$. Let $y, k$ be a code for $Y, K$, respectively. Clearly, $\langle y, k\rangle$ is a bad pair.

First, suppose the construction halted at stage $\alpha<\omega_{1}^{\mathrm{CK}}$. Let $z=\langle w, v, h\rangle$ be the unique $\alpha$-adequate real. The construction stops only if there does not exist a next bad pair which we have not picked already. Since $\langle y, k\rangle$ is a bad pair such that $k \subseteq^{*} h(n)$ for every $n \in \operatorname{dom}\left(<_{w_{\alpha}}\right)$, there must be an $n \in \operatorname{dom}\left(<_{w_{\alpha}}\right)$ such that $n=\langle y, k\rangle$, i.e., we picked $\langle y, k\rangle$ at that stage (otherwise, we can extend the construction by picking it now). But then $D_{v(n)}^{\omega^{\omega}} \cap D_{y}^{\omega^{\omega}}=\emptyset$, which implies $V \cap Y=\emptyset$ and so $V \cap X=\emptyset$.

Second, suppose the construction continued all the way up to $\omega_{1}^{\mathrm{CK}}$. Then there exists an $\alpha<\omega_{1}^{\mathrm{CK}}$ such that $\alpha>\varphi(\langle y, k\rangle)$. Let $z=\langle w, v, h\rangle$ be $\alpha$-adequate. 
By the observation above, the pair $\langle y, k\rangle$ was considered, hence there exists an $n \in \operatorname{dom}\left(<_{w_{\alpha}}\right)$ such that $n=\langle y, k\rangle$. Again, this implies $V \cap X=\emptyset$.

This finishes the proof of Proposition 5. We now derive a further refinement. A second key element of Harrington's proof is that $E$ is meager on $W \times W$, when $W$ is given the (subspace) Gandy-Harrington topology $\tau_{\mathrm{GH}}$. This is the topology on $\omega^{\omega}$ generated by the $\Sigma_{1}^{1}$ sets. Although $\omega^{\omega}$ with the Gandy-Harrington topology is not metrizable, it is strong Choquet and this enables one to redo the familiar construction of a perfect set of inequivalent elements, using a winning strategy for the second player. While this approach would also work in our case, we will use the set $X_{\text {low }}$ of low elements instead. This makes the construction in the proof of the main theorem more transparent, at the cost of some technicalities which we now summarize.

Let $X_{\text {low }}=\left\{x \in \omega^{\omega}: \omega_{1}^{\mathrm{CK}(x)}=\omega_{1}^{\mathrm{CK}}\right\}$. We will use the following facts about $W$, $X_{\text {low }}$, and $\tau_{\mathrm{GH}}$ :

1. $W$ and $X_{\text {low }}$ are both nonempty $\Sigma_{1}^{1}$ sets,

2. $X_{\text {low }}$ is dense in $\tau_{\mathrm{GH}}$ and $\left(X_{\mathrm{low}}, \tau_{\mathrm{GH}}\right)$ is a Polish space, and

3. a nonempty $\Sigma_{1}^{1}$ set $A \subseteq \omega^{\omega}$ meets uncountably many $E$-equivalence classes iff $A \cap W \neq \emptyset$ iff $A \cap W \cap X_{\text {low }} \neq \emptyset$.

Proofs of these facts can be found in [2].

Proposition 11. Let $E$ be a $\Pi_{1}^{1}$ equivalence relation on $\omega^{\omega}$ and $\left(A_{n}\right)_{n \in \omega}$ a se-

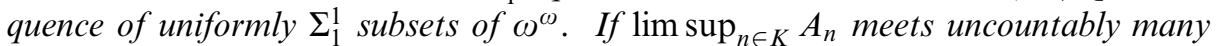
$E$-equivalence classes for every $K \in[\omega]^{\omega}$, then there exists a completely good pair $(V, H)$ such that $V$ is a Polish space in the Gandy-Harrington topology $\tau_{\mathrm{GH}}$ and $E$ is meager on $V \times V$ (with the product topology $\left.\tau_{\mathrm{GH}} \times \tau_{\mathrm{GH}}\right)$.

Proof. Let $(V, H)$ be the completely good pair given by Proposition 5 . Using the facts stated above, it is easy to see that $\left(V \cap W \cap X_{\text {low }}, H\right)$ is a completely good pair with the required additional properties.

§5. Proof of the main theorem. We now prove an effective version of Theorem 1 . By the usual relativization and transfer arguments, this implies our main result.

THEOREM 12. Let $E$ be a $\Pi_{1}^{1}$ equivalence relation on $\omega^{\omega}$ and $\left(A_{n}\right)_{n \in \omega}$ a sequence of uniformly $\Sigma_{1}^{1}$ subsets of $\omega^{\omega}$. If $\lim \sup _{n \in K} A_{n}$ meets uncountably many E-equivalence classes for every $K \in[\omega]^{\omega}$, then there exists a $K \in[\omega]^{\omega}$ such that $\bigcap_{n \in K} A_{n}$ contains a perfect set of pairwise $E$-inequivalent elements.

Proof. Let $(V, H)$ be the completely good pair given by Proposition 11 . Since $E$ is meager on $V \times V$ in the Gandy-Harrington topology $\tau_{\mathrm{GH}}$, we can fix an increasing sequence $\left(F_{n}\right)_{n \in \omega}$ of $\tau_{\mathrm{GH}}$-closed nowhere dense sets such that $E \subseteq \bigcup_{n \in \omega} F_{n}$. We may assume that the diagonal $\{(x, x): x \in V\}$ is contained in $F_{0}$. We will recursively define a strictly increasing sequence $j_{0}<j_{1}<\ldots$ of natural numbers and a Cantor scheme $\left(X_{s}\right)_{s \in 2^{<\omega}}$ of nonempty $\Sigma_{1}^{1}$ subsets of $V$ such that for all $s, t \in 2^{<\omega}$,

1. $\bar{X}_{s \frown 0}, \bar{X}_{s \frown 1} \subseteq X_{s}, \bar{X}_{s \frown 0} \cap \bar{X}_{s \frown 1}=\emptyset$, and $\operatorname{diam}\left(X_{s}\right) \leq 2^{-\operatorname{lh}(s)}$,

2. if $s \neq t \in 2^{n+1}$, then $X_{s} \times X_{t} \cap F_{n}=\emptyset$, and

3. if $s \in 2^{n}$, then $X_{s} \subseteq A_{j_{0}} \cap \cdots \cap A_{j_{n}}$. 
Note that in (1), the closures and diameter are relative to $\left(V, \tau_{\mathrm{GH}}\right)$. Once this construction is completed, let $K=\left\{j_{0}, j_{1}, \ldots\right\}$ and

$$
P=\bigcup_{\sigma \in 2^{\omega}} \bigcap_{n \in \omega} X_{\sigma\lceil n} .
$$

It is easy to see that $P \subseteq \bigcap_{n \in K} A_{n}$ is nonempty perfect set of pairwise $E$-inequivalent elements.

Without loss of generality we may assume that $A_{0}=\omega^{\omega}$. Start the construction with $j_{0}=0$ and $X_{\emptyset}=\omega^{\omega}$. Suppose we have defined natural numbers $j_{0}<\cdots<j_{n}$ and nonempty $\Sigma_{1}^{1}$ sets $X_{s} \subseteq A_{j_{0}} \cap \cdots \cap A_{j_{n}}$ for $s \in 2^{n}$ satisfying the requirements above. By intersecting with sufficiently small basic open neighborhoods, we can split each $X_{s}$ into disjoint nonempty $\Sigma_{1}^{1}$ sets $X_{s \sim 0}$ and $X_{s \sim 1}$ satisfying requirement (1). Since $F_{n}$ is closed nowhere dense, given any pair $s \neq t \in 2^{n+1}$ we can shrink $X_{s}$ and $X_{t}$ so that $X_{s} \times X_{t} \cap F_{n}=\emptyset$. After finitely many iterations, we have defined $X_{s}$ for $s \in 2^{n+1}$ satisfying requirements (1) and (2).

CLAim. There is $a j>j_{n}$ such that $X_{s} \cap A_{j} \neq \emptyset$ for all $s \in 2^{n+1}$.

Proof. Suppose towards a contradiction that for every $j>j_{n}$ there is an $s \in 2^{n+1}$ such that $X_{s} \cap A_{j}=\emptyset$. Define a binary relation $R \subseteq \omega \times 2^{n+1}$ by $R(j, s) \Leftrightarrow$ $X_{s} \cap A_{j}=\emptyset$. Since $R$ is $\Pi_{1}^{1}$, there is a $\Delta_{1}^{1}$ uniformizing function $f: \omega \rightarrow 2^{n+1}$. By the pigeonhole principle, there is an $s \in 2^{n+1}$ such that $\{j \in \omega: f(j)=s\} \cap H$ is infinite. Pick such an $s \in 2^{n+1}$. Then $K=\{j \in \omega: j \in H$ and $f(j)=s\}$ is $\Delta_{1}^{1}$, $K \in[H]^{\omega}$, and $X_{s} \cap \bigcup_{n \in K} A_{n}=\emptyset$. This implies that $X_{s} \cap \lim \sup _{n \in K} A_{n}=\emptyset$, contradicting the fact that $(V, H)$ is a completely good pair.

To complete this step in the construction, let $j_{n+1}=j$ and intersect each $X_{s}$ with $A_{j_{n+1}}$. This finishes the proof of Theorem 12 .

The following parametric version of the Laczkovich-Komjáth property was also considered by Balcerzak and Głạb.

Definition. An equivalence relation $E$ on a Polish space $Y$ has the parametric Laczkovich-Komjáth property if for every uncountable Polish space $X$ and every sequence $\left(A_{n}\right)_{n \in \omega}$ of analytic subsets of $X \times Y$, if $\lim _{\sup } \operatorname{su}_{n \in K} A_{n}(x)$ meets uncountably many $E$-equivalence classes for every $x \in X$ and $K \in[\omega]^{\omega}$, then there exists a $K \in[\omega]^{\omega}$ and a perfect set $P \subseteq X$ such that $\bigcap_{n \in K} A_{n}(x)$ meets perfectly many $E$-equivalence classes for each $x \in P$.

Theorem 13 (Balcerzak-Głąb [1]). If E has the Laczkovich-Komjáth property and for every analytic set $A \subseteq X \times X$, the set

$\left\{x \in X: A_{x}\right.$ meets uncountably many E-equivalence classes $\}$

is analytic, then $E$ has the parametric Lackovich-Komjáth property.

PROPOSITION 14. Every coanalytic equivalence relation on a Polish space has the parametric Laczkovich-Komjáth property.

Proof. Let $E$ be a coanalytic equivalence relation on a Polish space $X$ and $A \subseteq X \times X$ an analytic subset. Without loss of generality we may assume $E$ is a $\Pi_{1}^{1}$ equivalence relation on $X=\omega^{\omega}$ and $A \subseteq \omega^{\omega} \times \omega^{\omega}$ is $\Sigma_{1}^{1}$. Since $A$ is $\Sigma_{1}^{1}$, each section $A_{x}$ is $\Sigma_{1}^{1}$ as well. Hence, $A_{x}$ meets uncountably many $E$-equivalence classes 
iff $A_{x} \cap W \neq \emptyset$. Thus,

$\left\{x \in \omega^{\omega}: A_{x}\right.$ meets uncountably many $E$-equivalence classes $\}$

is $\Sigma_{1}^{1}$. Hence, $E$ has the parametric Laczkovich-Komjáth property by Theorem 1 and Theorem 13.

\section{REFERENCES}

[1] Marek BalcerzaK and Szymon GląB, On the Laczkovich-Komjáth property of sigma-ideals, preprint.

[2] Su GaO, Invariant descriptive set theory, Taylor \& Francis Group, 2009.

[3] Su Gao, Steve Jackson, Miklós Laczkovich, and R. Daniel Mauldin, On the unique representation of families of sets, Transactions of the American Mathematical Society, vol. 360 (2008), no. 2, pp. 939-958.

[4] Leo A. Harrington, Alexander S. Kechris, and Alain louveau, A Glimm-Effros dichotomy for Borel equivalence relations, Journal of the American Mathematical Society, vol. 3 (1990), no. 4. pp. 903-928.

[5] Peter КомjÁth, On the limit superior of analytic sets, Analysis Mathematica, vol. 10 (1984), no. 4, pp. 283-293.

[6] Miklós Laczkovich, On the limit superior of sequences of sets, Analysis Mathematica, vol. 3 (1977), no. 3, pp. 199-206.

[7] Donald A. Martin and Alexander S. Kechris, Infinite games and effective descriptive set theory, Analytic sets, Academic Press, 1980.

[8] Yiannis N. Moschovakis, Descriptive set theory, Studies in Logic and the Foundations of Mathematics, no. 100, North-Holland Publishing Company, 1980.

[9] JACK H. SILvER, Counting the number of equivalence classes of Borel and coanalytic equivalence relations, Annals of Mathematical Logic, vol. 18 (1980), no. 1, pp. 1-28.

UNIVERSITY OF NORTH TEXAS

DEPARTMENT OF MATHEMATICS

1155 UNION CIRCLE \#311430

DENTON, TX 76203-5017, USA

E-mail:sgao@unt.edu

E-mail: jackson@unt.edu

E-mail: kieftenbeld@unt.edu 